\title{
Etude de la productivité des parcours de la zone d'Ain-Leuh (Moyen Atlas, plateau central). I. Effets de la fréquence d'exploitation et du taux de couvert arboré sur la productivité herbacée
}

\author{
M. Qarro ${ }^{1}$ et F.X. de Montard² \\ 1 Ecole Nationale Forestière d'Ingénieurs, B.P. 511, Tabriquet-Salé, Maroc \\ 2INRA, Station d'Agronomie, 12, avenue du Brézet, F 63039 Clermont-Ferrand, France
}

(reçu le 13 octobre 1988, accepté le 16 février 1989)

Résumé - Les parcours à moutons du Moyen Atlas sont étagés de 900 à $2200 \mathrm{~m}$ d'altitude sur quatre formations : chênaie verte à oxycèdre, chênaie verte pure, cédraie et pelouse asylvatique. Une étude de la productivité de la strate herbacée à été menée en 1983 et 1984 pour comparer l'effet de différents intervalles entre coupes successives. La pousse de l'herbe a lieu principalement entre janvier et juillet; elle est retardée par le froid aux étages de la cédraie et de la pelouse, et ralentie précocement par la sécheresse aux étages de la chênaie.

La productivité moyenne est de 2 à $3 \mathrm{~kg}$ de M.S. ha-1 $\mathrm{j}^{-1}$ sur les sols squelettiques de la chênaie à oxycèdre, et sur les sols dolomitiques de la cédraie; elle est de 5 à $6 \mathrm{~kg} \mathrm{ha}^{-1} \mathrm{j}^{-1}$ sur les sols d'éboulis de la chênaie verte pure et le sol basaltique de la pelouse. Les différences de production entre saisons et entre étages sont expliquées principalement par le niveau d'alimentation en eau et l'abondance des légumineuses.

Le rythme de coupe le plus favorable est 4 coupes par an; 3 ou 5 coupes par an produisent encore 75 à $90 \%$ du rythme 4 coupes; l'absence de coupe précoce entraîne l'inhibition des organes de repousse végétative et une réduction de la productivité annuelle, de moitié avec deux coupes, des $3 / 5^{\Theta}$ avec une seule coupe.

L'ombrage des arbres ne réduit pas ou peu la productivité de la strate herbacée jusqu'à $60 \%$ de couvert arboré: a) lorsque l'alimentation en eau n'est pas limitante, la productivité baisse d'un tiers sous un couvert de 35 à $65 \%$; b) lorsqu'une sécheresse relative intervient, la productivité n'est pas affectée par l'ombrage. Au-delà de $70 \%$, la productivité de la strate herbacée baisse très rapidement.

Un aménagement sylvo-pastoral réalisant entre 60 et $70 \%$ de couvert arboré constituerait donc un objectif très favorable à la cohabitation de l'élevage et de la production de bois.

matière sèche - déficit hydrique - rythme - coupe - sylvo-pastoral

Summary - A study of dry matter production in rough pastures of Ain-Leuh (Middle Atlas, central plateau). I. Effects of cut frequency and relative tree cover on herbaceous production. Rough pastures in Middle Atlas spread over four bioclimatic levels: 1, Quercus ilex and Juniperus oxycedrus forest; 2, pure Quercus ilex forest; 3. Cedrus atlantica and Quercus ilex forest; 4, unsylvatic low grass, from 900 to $2200 \mathrm{~m}$ above sea level. Dry matter production of grass was measured from and underneath the tree canopy in 1983 and 1984 in order to compare the effect of a variety of intervals between cuts and to measure shadow influence. Grass growth mainly occured from late January to early July; it began later at Cedrus-Quercus and low grass sites and stopped earlier at pure Quercus and Quercus-Juniperus sites, depending on longer frost persistence at highest levels, on earlier arrival of drought at lowest levels.

Mean dry matter production was $2.5 \mathrm{~kg} \mathrm{ha}^{-1} \mathrm{~d}^{-1}$ on light $30 \mathrm{~cm}$ deep soils at Quercus-Juniperus level and dolomitic soils at Cedrus-Quercus level. It was $5.5 \mathrm{~kg} \mathrm{ha}^{-1} \mathrm{~d}^{-1}$ on colluvial soils at pure Quercus level and basaltic soil at lowgrass level. Water balance and legume species abundance explained a great part of D.M. variance.

Four cuts per year were more productive than three or five. Missing the earliest cuts resulted in much lower D.M. yields: a half with two cuts; three of five with an only cut per year.

Shadow poorly reduced the yield up to a forest canopy cover of about $60 \%$ : from 35 to $65 \%$ cover, the canopy resulted in $30 \%$ fall in seasonal yield as water supply was not limiting, but had no effect as relative water deficit occurred. A pasture and forestry mixed system, aiming a canopy of 60 to $70 \%$ cover, would result in better conditions for cattle breeding and timber as a whole. 


\section{Introduction}

Les aires de parcours des troupeaux, ovins et caprins, des tribus du Moyen Atlas sont constituées essentiellement de quatre types de formations végétales : chênaie verte à oxycèdre en climat semi-aride du plateau central, chênaie verte pure en climat subhumide du Dir, cédraie à sousétage de chêne vert et pelouse asylvatique des montagnes mésatlasiques en climat humide à variantes froide à très froide.

Etagées le long d'un gradient altitudinal pluvial et thermique, ces formations jouent un rôle complémentaire au cours du cycle pastoral annuel. Cette complémentarité en fait un ensemble cohérent, unifié par les pratiques pastorales des tribus usagères.

La croissance de l'herbe au cours du cycle annuel varie avec l'étage bioclimatique et les conditions saisonnières:

- dans le semi-aride et le subhumide, un certain reverdissement peut avoir lieu d'octobre à janvier en fonction des pluies qui restent irrégulières à cette époque à ces altitudes. La croissance et le développement commencent en réalité vers janvier et février pour aboutir à la floraison en avril et mai;

- à l'étage de l'humide, variantes froide à très froide, la reprise d'automne serait plutôt mie:sx assurée par un bilan hydrique plus favorable, si la pousse n'était stoppée ou ralentie par les températures basses. Après un faux départ à l'automne, le redémarrage de la végétation de février à mars aboutit à la floraison vers mai et juin;

- juin et juillet couvrent la période du dessèchement, assez variable selon l'année et l'étage (Fig. $1 a, b, c, d)$ : toujours précoce, vers fin mai, voire courant juin, à l'étage de la chênaie verte à oxycèdre, et plus tardive, vers juin et juillet selon l'élévation en altitude, de la chênaie verte du Dir à la pelouse asylvatique.

La production en matière sèche de la strate herbacée de ces parcours boisés a généralement été sous-estimée parce que les mesures étaient faites dans de mauvaises conditions, trop tardives et sur un faible nombre annuel de coupes, sans rapport avec l'effet du broutage précoce et répété des ovins. Différents auteurs ont essayé de résoudre ce problème de mesure (Milner et Elfynhughes, 1968; Gounot et Yu 1980; Levang et Grouzis, 1980; Cornet, 1981).

Cette production varie aussi énormément en fonction des caractéristiques de la station et des variations climatiques inter ou intra-annuelles (Qarro, 1985).

Pour parvenir à un meilleur compromis entre la production forestière et les usages pastoraux,
0)

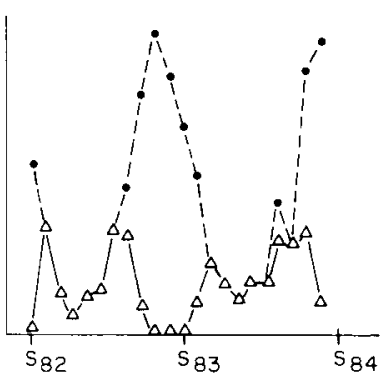

c)

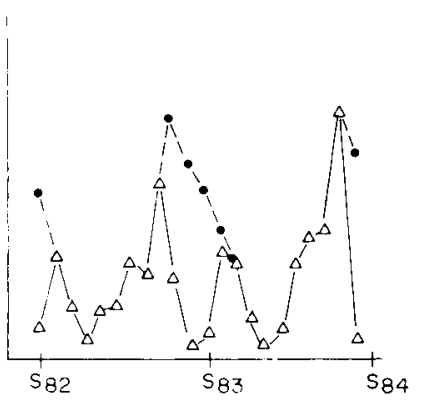

b)

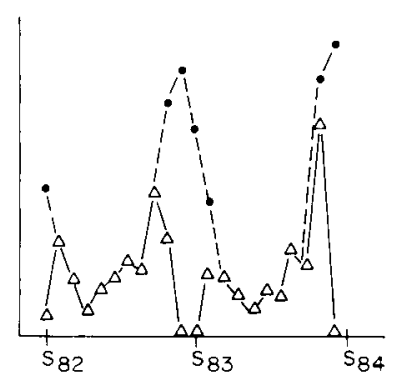

d)

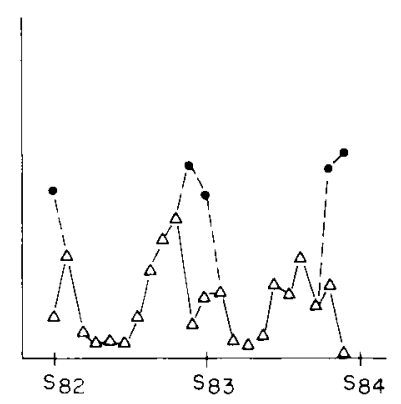

Fig. 1. Evolution de l'évapotranspiration potentielle et de l'évapotranspiration calculée d'après le bilan hydrique : $(\bullet--\cdots)$ ETP; $(\Delta-\Delta)$ ET calculée en $\mathrm{mm}$ par mois ou par période; successivement : sept., oct., nov., déc. 1982; jv., fév., P2, P3, P4, P5, août, sept., oct., nov., déc. 1983; jv., fév., $P 2, P 3, P 4, P 5$, août 1984. $S_{82}, S_{83}, S_{84}$ : bornes de septembre à septembre. a) Chênaie à oxycèdre; b) Chênaie verte pure; c) Cédraie; d) Pelouse.

il est primordial de connaître les potentialités herbagères réelles de ces parcours et, surtout, la réponse spécifique de la strate herbacée aux facteurs écologiques (Christie, 1978).

L'objet de cette étude est la mesure de la productivité de la strate herbacée en fonction du rythme de coupe et des facteurs écologiques liés au climat, au sol et au couvert arboré, et un essai de modélisation simple (Saugier, 1982).

\section{Matériels et Méthodes}

La zone étudiée s'étend à cheval sur le plateau central et les montagnes du Moyen Atlas, sur le territoire de la commune rurale d'Ain-Leuh; elle comprend quatre milieux écologiquement et géographiquement différents (Qarro, 1985) :

- la chênaie verte a oxycèdre dégradée du plateau central. L'action destructive de l'homme et des troupeaux y est accentuée par les conditions climatiques semi-arides et le caractère squelettique des sols sur schistes;

- la chênaie verte pure couvre le Dir, zone de rupture qui fait la jonction entre le plateau central et les montagnes du Moyen Atlas. Elle constitue des peuplements bien venants de chêne vert établis sur des argiles rouges du Trias et parfois sur des affleurements dolomitiques. Le bioclimat subhumide de cette zone serait favorable à la végétation si la pression humaine était plus modérée; mais ce secteur est par- 
semé d'habitats permanents et constitue le point de départ de tous les troupeaux:

- les formations de cèdre couvrent les montagnes mésatlasiques sous climat humide à variante froide; ces formations comportent un sous-étage de chênes verts qui disparaît progressivement avec l'altitude et aux expositions fraîches;

- les pelouses occupent les zones asylvatiques de l'étage supérieur, dans les cuvettes gélives, peu propices à la végétation arborée. Ces milieux constituent une réserve importante pour l'alimentation du cheptel de la zone qui s'y concentre en été; ils reçoivent beaucoup de neige. Le bioclimat y est une variante très froide de l'humide.

L'étude a porté sur quatre stations, Tourtit, Tizemourine, Kissarit et Aïn Kahla, représentant, dans l'ordre d'altitude croissante, les différents milieux de la zone. Ces stations ont été encloses pour les exclure du parcours et permettre les mesures de production en matière sèche de la strate herbacée. En fonction des facteurs de l'environnement, un certain nombre de sous-stations ont été repérées dans chacune d'elles (Tableau I). A l'intérieur de la dominante physionomique générale propre à chacun des quatre types de milieux, les sous-stations représentent les différents faciès décrits par le taux de recouvrement arboré (projection du couvert arboré sur la voûte céleste, mesurée au densiomètre), l'exposition et la pente, et disposés en mosaïque sur des surfaces homogènes de quelques centaines de $\mathrm{m}^{2}$.

Au total, 24 sous-stations ont été définies comme suit :

- 9 sous-stations dans la chênaie verte à oxycèdre;

-8 sous-stations dans la chênaie verte pure;

- 6 sous-stations dans la cédraie à sous-étage de chênes verts;

- 1 seule dans la pelouse asylvatique.

Dans chaque sous-station, 20 placettes $\mathrm{d}^{\prime} 1 \mathrm{~m}^{2}$ ont été délimitées dans un rectangle de $10 \mathrm{~m} \times 2 \mathrm{~m}$, aussi homogène que possible. A chacune des cinq visites effectuées dans l'année, plusieurs placettes passent à la coupe : ainsi, 10 modalités d'échelonnement des coupes ont été définies au préalable et disposées selon un organigramme randomisé (2 placettes d'1 $\mathrm{m}^{2}$ par modalité). L'étude a été limitée à la période qui s'étend de novembre à juillet et couvre l'essentiel de la croissance herbacée.

Tableau I. Description des sous-stations : facteurs de l'environnement.

\begin{tabular}{|c|c|c|c|c|c|c|c|c|c|}
\hline Formation & $\begin{array}{l}\text { S/Sta- } \\
\text { tion }\end{array}$ & $\begin{array}{l}\text { Couvert } \\
\text { arboré }\end{array}$ & Exposition & $\begin{array}{l}\text { Pente } \\
\%\end{array}$ & Roche & $\begin{array}{l}\text { Profon- } \\
\text { deur }\end{array}$ & $\begin{array}{l}\text { M.O. } \\
\%\end{array}$ & \% Kéch. & $\begin{array}{l}\text { Légumi- } \\
\text { neuses }\end{array}$ \\
\hline Chênaie & 1 & * 0 & $S$ & 30 & Schiste & 37 & 2,37 & 0,32 & 0 \\
\hline \multirow[t]{8}{*}{ à oxycèdre } & 4 & 0 & $\mathrm{~S}$ & 15 & Schiste & 30 & 2,27 & 0,19 & 1 \\
\hline & 5 & 0 & $S$ & 30 & Grès & 30 & 1,85 & 0,12 & 11 \\
\hline & 9 & * 0 & W & 20 & Schiste & 30 & 1,75 & 0,19 & 12 \\
\hline & 7 & 13 & replat & 3 & Schiste & 30 & 2,37 & 0,25 & 10 \\
\hline & 3 & * 19 & $W$ & 10 & Schiste & 29 & 2,27 & 0,25 & 13 \\
\hline & 2 & 33 & W & 20 & Schiste & 34 & 1,23 & 0,25 & 3 \\
\hline & 6 & 62 & W & 30 & Grès & 38 & 2,88 & 0,12 & 2 \\
\hline & 8 & 70 & $\mathrm{~N}$ & 45 & Schiste & 25 & 2,06 & 0,12 & 3 \\
\hline \multirow{8}{*}{$\begin{array}{l}\text { Chênaie } \\
\text { pure }\end{array}$} & 12 & 0 & replat & 5 & Calcaire & 40 & 7,74 & 1,24 & 28 \\
\hline & 13 & 0 & $w$ & 25 & Calcaire & 38 & 8,04 & 0,96 & 12 \\
\hline & 14 & 19 & replat & 0 & Calcaire & 33 & 13,78 & 1,66 & 46 \\
\hline & 16 & 20 & $E$ & 8 & Calcaire & 50 & 5,36 & 0,44 & 11 \\
\hline & 11 & ** 38 & $S$ & 18 & Calcaire & 50 & 6,29 & 1,28 & 75 \\
\hline & 17 & ** 39 & W & 21 & Calcaire & 40 & 10,01 & 1,02 & 62 \\
\hline & 10 & 62 & $w$ & 18 & Calcaire & 30 & 5,67 & 1,15 & 47 \\
\hline & 15 & 91 & $\mathrm{~N}$ & 55 & Calcaire & 50 & 34,20 & 1,15 & 0 \\
\hline \multirow[t]{6}{*}{ Cédraie } & 21 & 0 & replat & 0 & Dolomie & 30 & 5,67 & 0,83 & 15 \\
\hline & 23 & 0 & replat & 0 & Dolomie & 60 & 8,35 & 1,21 & 2 \\
\hline & 20 & 65 & replat & 0 & Dolomie & 55 & 19,50 & 1,28 & 1 \\
\hline & 18 & 70 & replat & 0 & Dolomie & 30 & 9,18 & 1,08 & 6 \\
\hline & 19 & 75 & replat & 0 & Dolomie & 75 & 7,74 & 1,08 & 6 \\
\hline & 22 & 83 & replat & 0 & Dolomie & 55 & 19,50 & 1,28 & 1 \\
\hline Pelouse & 24 & 0 & replat & 4 & Basalte & 55 & 9,18 & 2,31 & 16 \\
\hline
\end{tabular}

"s/st. 1 : sol sableux (74\% de sable grossier); s/st. 3 : sol grossier sur sommet; s/st. 9 située dans un vallonnement.

"Anthyllis vulneraria abondant : Abondance-Dominance supérieure à 4. 
Cinq dates de coupes ont été retenues chaque année : la première intervient vers la fin février; les autres suivent tous les mois : 1 re semaine d'avril, $1^{\text {re }}$ semaine de mai, $1^{\text {re }}$ semaine de juin; la dernière date est retardée jusqu'à la $3^{e}$ semaine de juillet, moment où débute généralement le dessèchement. La chênaie verte à oxycèdre a fait exception en 1983, aucune repousse n'étant intervenue après la coupe de juin, par suite d'une sécheresse précoce.

Chaque placette d' $1 \mathrm{~m}^{2}$ subit, en fonction de l'organigramme des coupes déterminé à l'avance, un nombre de coupes différent, variant de 1 à 5 . Ces décalages entre coupes successives ont permis de suivre l'évolution de la productivité des différentes sous-stations en fonction d'intervalles variés par leur longueur et leur position dans la saison de végétation, les "périodes". 13 périodes ont été étudiées en combinant les 5 dates de coupes dans la saison de février à juillet; ( 9 périodes seulement en 1983, dans le cas de la chênaie à oxycèdre). Ces périodes ont été définies de la même façon pour les 4 étages bioclimatiques de manière à faciliter les comparaisons :

P1 est la période comprise entre le redémarrage de la végétation et la fin février; avril;

P2 est la période comprise entre fin février et début

P3 entre début avril et début mai;

P4 entre début mai et début juin;

P5 entre début juin et la troisième semaine de juillet;

P6 entre la reprise de végétation et début avril;

$P 7$ entre la reprise de végétation et début mai;

P8 entre la reprise de végétation et début juin;

P9 entre la reprise et la troisième semaine de juillet;

P10 entre fin février et début mai;

P11 entre fin février et début juin;

$P 12$ entre début avril et début juin;

P13 entre début mai et la troisième semaine de juillet.

Ce protocole a été appliqué sur deux années agricoles successives, mais contrastées par le régime pluviométrique : 1982-1983 est une année à sécheresse précoce, et 1983-1984 une année humide; un tel contraste est particulièrement satisfaisant vis-à-vis de l'objectif de cette expérimentation compte tenu des obstacles pratiques et théoriques à la poursuite des mesures sur les mêmes sites au-delà de deux ans.

\section{Résultats et discussion}

Une étude comparée des quatre milieux s'impose du fait qu'ils constituent les éléments d'un même ensemble pastoral, parcourus progressivement et en complémentarité au cours du cycle annuel.

D'autre part, au fur et à mesure de l'établissement des tableaux des résultats par étage, des réponses similaires ont pu être relevées, rendant souhaitable une interprétation plus générale intégrant les quatre milieux.
Calcul de productivité et classement des périodes

Pour faciliter la comparaison des résultats de production obtenus sur des périodes de temps de longueur variable, les productivités moyennes journalières par période ont été calculées (productivité exprimée en $\mathrm{kg} \mathrm{ha}^{-1} \mathrm{j}^{-1}$ ). Une première analyse simple a permis de regrouper les résultats selon trois types de périodes:

- les périodes «indéterminées», qui commencent au démarrage de la végétation en automne, $\mathrm{P} 1$, $\mathrm{P} 6, \mathrm{P} 7, \mathrm{P} 8$ et $\mathrm{P9}$, et couvrent quatre mois et plus avant que la première coupe intervienne (Tableau II) (le calcul de productivité est opéré en prenant le début novembre comme date initiale, c'est-à-dire, pour les deux années considérées, à partir de la date de reconstitution de la réserve en eau des sols);

- les périodes "déterminées" (Tableau III) : a) "simples", P2, P3, P4 et P5, couvrent 1 mois environ entre deux coupes (6 semaines pour $\mathrm{P} 5)$; b) "doubles ou triples" couvrent deux mois (P10, P12, P13) ou trois mois environ (P11).

En 1983, la sécheresse précoce a empêché la réalisation des périodes $\mathrm{P} 5, \mathrm{P} 9, \mathrm{P} 11$ et $\mathrm{P} 13$ à l'étage du semi-aride.

Effets de la date et du rythme de coupe : résultats de productivité en matière sèche par période

\section{Productivité des périodes indéterminées}

D'après les résultats obtenus sur ces deux années d'expérimentation (Tableaux II et III), les périodes indéterminées ont une productivité beaucoup plus faible que celle des périodes déterminées, indépendamment du couvert arboré : $28 \%$ de celle des périodes déterminées dans le semi-aride, 35 à $40 \%$ dans le subhumide et l'humide frais, 35 à $50 \%$ dans l'humide froid, soit $36 \%$ en moyenne pour l'ensemble des 4 stations.

Ce phénomène est particulièrement marqué sur les sols squelettiques de la chênaie à oxycèdre (moins de $1 \mathrm{~kg} \mathrm{ha}^{-1} \mathrm{j}^{-1}$, en général).

Cette faible productivité est la résultante de deux phénomènes:

- la faible croissance observée en jours courts avec des températures modérées dans le semiaride et le subhumide et même froides en décembre et janvier dans l'humide froid, en novembre, décembre et janvier dans l'humide très froid. La sécheresse peut encore intervenir à cette période dans le semi-aride certaines années.

- l'arrêt précoce de la croissance de l'herbe lorsque les tiges reproductrices ne sont pas sup- 
Tableau II. Productivité des périodes indéterminées en matière sèche.

\begin{tabular}{|c|c|c|c|c|c|c|c|}
\hline Mois & & $\begin{array}{c}\text { Nv à Fe } \\
P 1\end{array}$ & $\begin{array}{c}N v \text { à Ma Nv à } A v \\
P 6\end{array}$ & $\begin{array}{l}N v \text { à } M i N v \text { à } J t \\
\quad P 7\end{array}$ & $\begin{array}{l}\text { Moyenne } \\
\text { P8 }\end{array}$ & $P 9$ & \\
\hline $\begin{array}{l}\text { Chênaie verte } \\
\text { à oxycèdre }\end{array}$ & $\begin{array}{l}83 \\
84\end{array}$ & $\begin{array}{l}0,53 \\
0,20\end{array}$ & $\begin{array}{l}0,60 \\
0,65\end{array}$ & $\begin{array}{l}0,61 \\
0,80\end{array}$ & $\begin{array}{l}0,73 \\
1,92\end{array}$ & - & $\begin{array}{l}0,62 \\
0,89\end{array}$ \\
\hline $\begin{array}{l}\text { Chênaie verte } \\
\text { pure }\end{array}$ & $\begin{array}{l}83 \\
84\end{array}$ & $\begin{array}{l}0,84 \\
0,93\end{array}$ & $\begin{array}{l}1,30 \\
1,46\end{array}$ & $\begin{array}{l}1,91 \\
2,17\end{array}$ & $\begin{array}{l}2,68 \\
3,77\end{array}$ & $\begin{array}{l}1,01 \\
1,84\end{array}$ & $\begin{array}{l}1,55 \\
2,03\end{array}$ \\
\hline Cédraie & $\begin{array}{l}83 \\
84\end{array}$ & $\begin{array}{l}0,71 \\
0,32\end{array}$ & $\begin{array}{l}0,76 \\
0,57\end{array}$ & $\begin{array}{l}0,90 \\
0,86\end{array}$ & $\begin{array}{l}1,54 \\
1,40\end{array}$ & $\begin{array}{l}0,65 \\
0,75\end{array}$ & $\begin{array}{l}0,91 \\
0,78\end{array}$ \\
\hline $\begin{array}{l}\text { Pelouse } \\
\text { asylvatique }\end{array}$ & $\begin{array}{l}83 \\
84\end{array}$ & $\begin{array}{l}1,10 \\
0,67\end{array}$ & $\begin{array}{l}2,94 \\
0,94\end{array}$ & $\begin{array}{l}3,60 \\
1,56\end{array}$ & $\begin{array}{l}8,05 \\
3,83\end{array}$ & $\begin{array}{l}0,64 \\
2,05\end{array}$ & $\begin{array}{l}3,27 \\
1,80\end{array}$ \\
\hline
\end{tabular}

Tableau Ill. Productivité des périodes déterminées en matière sèche.

\begin{tabular}{|c|c|c|c|c|c|c|c|c|c|c|}
\hline $\begin{array}{l}\text { Durée } \\
\text { Mois } \\
\text { Périodes }\end{array}$ & & $\begin{array}{l}\text { MARS } \\
\text { P2 }\end{array}$ & $\begin{array}{l}1 \mathrm{~m} \\
\text { AVR } \\
\text { P3 }\end{array}$ & $\begin{array}{l}\text { is } \\
\text { MAI }\end{array}$ & $\begin{array}{l}J \& J \\
P 5\end{array}$ & $\begin{array}{l}2 r \\
M \& A \\
P 10\end{array}$ & $\begin{array}{l}\text { A\&M } \\
P 12\end{array}$ & $\begin{array}{l}M_{J J}^{3} \\
P 13\end{array}$ & $\begin{array}{l}\text { is } \\
\text { MAM }\end{array}$ & Moyenne \\
\hline $\begin{array}{l}\text { Ch. verte } \\
\text { à oxycèdre }\end{array}$ & $\begin{array}{l}83 \\
84\end{array}$ & $\begin{array}{l}3,11 \\
1,81\end{array}$ & $\begin{array}{l}2,42 \\
4,73\end{array}$ & $\begin{array}{l}2,29 \\
4,94\end{array}$ & $\overline{1,09}$ & $\begin{array}{l}1,97 \\
2,02\end{array}$ & $\begin{array}{l}1,69 \\
5,07\end{array}$ & $\overline{1,38}$ & $\overline{3,51}$ & $\begin{array}{l}2,30 \\
3,06\end{array}$ \\
\hline $\begin{array}{l}\text { Ch. verte } \\
\text { pure }\end{array}$ & $\begin{array}{l}83 \\
84\end{array}$ & $\begin{array}{l}6,64 \\
2,85\end{array}$ & $\begin{array}{l}5,15 \\
4,81\end{array}$ & $\begin{array}{l}4,82 \\
6,94\end{array}$ & $\begin{array}{l}1,77 \\
1,76\end{array}$ & $\begin{array}{l}5,43 \\
4,61\end{array}$ & $\begin{array}{l}4,58 \\
9,92\end{array}$ & $\begin{array}{l}1,18 \\
2,16\end{array}$ & $\begin{array}{l}5,47 \\
6,43\end{array}$ & $\begin{array}{l}4,38 \\
4,93\end{array}$ \\
\hline Cédraie & $\begin{array}{l}83 \\
84\end{array}$ & $\begin{array}{l}2,52 \\
1,23\end{array}$ & $\begin{array}{l}3,42 \\
2,75\end{array}$ & $\begin{array}{l}2,73 \\
2,48\end{array}$ & $\begin{array}{l}1,05 \\
1,28\end{array}$ & $\begin{array}{l}3,06 \\
2,04\end{array}$ & $\begin{array}{l}3,38 \\
3,64\end{array}$ & $\begin{array}{l}0,75 \\
1,68\end{array}$ & $\begin{array}{l}3,00 \\
2,09\end{array}$ & $\begin{array}{l}2,48 \\
2,15\end{array}$ \\
\hline $\begin{array}{l}\text { Pelouse } \\
\text { asylvatique }\end{array}$ & $\begin{array}{l}83 \\
84\end{array}$ & $\begin{array}{l}4,84 \\
2,76\end{array}$ & $\begin{array}{l}12,0 \\
4,40\end{array}$ & $\begin{array}{l}8,09 \\
7,13\end{array}$ & $\begin{array}{l}2,91 \\
4,05\end{array}$ & $\begin{array}{l}5,83 \\
3,18\end{array}$ & $\begin{array}{r}9,02 \\
10,42\end{array}$ & $\begin{array}{l}0,88 \\
4,73\end{array}$ & $\begin{array}{l}9,76 \\
5,07\end{array}$ & $\begin{array}{l}6,66 \\
5,22\end{array}$ \\
\hline
\end{tabular}

primées assez tôt, et la sénescence de la végétation non exploitée.

Le premier phénomène est évident. Pour le second, l'examen de la productivité de la période P6 (novembre à mars inclus) indique des valeurs presque toutes inférieures à la moitié de la productivité de P8 (novembre à mai inclus); ceci montre qu'un certain rattrapage s'effectue lors de la montaison en avril et mai. La productivité de la période $\mathrm{P} 8$ se rapproche ainsi du niveau moyen de productivité des périodes les plus favorables, $\mathrm{P} 2, \mathrm{P} 3, \mathrm{P} 4, \mathrm{P} 10, \mathrm{P} 12$ et $\mathrm{P} 11$ qui couvrent respectivement 1,2 ou 3 mois de la période mars-avrilmai. Ce rattrapage est fonction de l'humidité du climat, de températures moyennes favorables et de la fertilité du milieu (Tableau IV).

Une comparaison complète nécessite un examen des productions cumulées sur l'année avec les différents régimes de coupe appliqués (Tableau V) : dans les 4 milieux, la plus forte production est obtenue avec 4 coupes. Aux étages supérieurs, plus humides et plus frais, les productions annuelles obtenues avec 3 ou 5 coupes sont assez proches de celle de 4 coupes; mais, en revanche, avec le déficit hydrique accentué des 2 étages inférieurs, l'écart se creuse (diminu- tion de 20 à 25\%). La production obtenue avec deux coupes n'est plus que la moitié ou les trois quarts de la production obtenue avec quatre, et avec une coupe unique, seulement un tiers ou la moitié.

\section{Productivité des périodes déterminées}

Une autre cause de baisse de productivité apparaît nettement à l'examen des résultats des périodes déterminées (Tableau III). Les périodes qui incluent la phase du déssèchement, qui survient parfois dès juin mais le plus souvent en juillet, extériorisent une productivité particulièrement faible, quelle que soit leur longueur (P5 1 mois et demi, P13 mai à juillet et, pour mémoire, la période indéterminée P9 qui s'étend de novembre à juillet).

- La période P5 exprime directement l'effet du déficit hydrique.

- La période P13, qui devrait extérioriser une productivité intermédiaire entre $\mathrm{P} 4$ (mai) et $\mathrm{P} 5$ (juin et juillet), est aussi faible que P5 : il y a donc, par temps sec, une perte importante de la matière sèche accumulée en mai et début juin, soit par chute dans la litière, soit par consommation par les acridiens. 
Tableau IV. Rapport de la productivité de la période indéterminée P8 à la productivité moyenne des périodes déterminées correspondantes.

[Productivité P8 / Productivité moyenne $(\mathrm{P} 2,3,4,10,12,11)] \times 100$

\begin{tabular}{llll}
\hline $\begin{array}{l}\text { Chênaie à oxycèdre } \\
\text { (milieu pauvre) }\end{array}$ & année 1 & (très sèche) & $32 \%$ \\
Chênaie verte pure & année 2 & (humide) & $52 \%$ \\
(milieu riche) & année 1 & (sèche) & $50 \%$ \\
Cédraie & année 2 & (humide) & $64 \%$ \\
(milieu peu productif) & année 1 & (sèche) & $51 \%$ \\
Pelouse & année 2 & (humide) & $59 \%$ \\
(milieu riche) & année 1 & (humide) & $97 \%$ \\
\hline
\end{tabular}

Tableau V. Production annuelle de matière sèche en fonction du nombre de coupes (moyenne des 2 années en $\mathrm{kg}$ $\mathrm{ha}^{-1} \mathrm{an}^{-1}$ et en indice \%).

\begin{tabular}{|c|c|c|c|c|c|c|c|c|}
\hline \multirow[b]{2}{*}{$N b$} & \multicolumn{2}{|c|}{$\begin{array}{l}\text { Chênaie verte } \\
\text { à oxycèdre }\end{array}$} & \multicolumn{2}{|c|}{$\begin{array}{c}\text { Chênaie verte } \\
\text { pure }\end{array}$} & \multicolumn{2}{|c|}{ Cédraie } & \multicolumn{2}{|c|}{ Pelouse } \\
\hline & $\mathrm{kg}$ & $\%$ & $\mathrm{~kg}$ & $\%$ & $\mathrm{~kg}$ & $\%$ & $\mathrm{~kg}$ & $\%$ \\
\hline 1 & 169 & 53 & 344 & 48 & 177 & 51 & 331 & 34 \\
\hline 2 & 220 & 69 & 505 & 71 & 261 & 75 & 541 & 53 \\
\hline 3 & 248 & 78 & 544 & 76 & 326 & 93 & 799 & 83 \\
\hline 4 & 320 & 100 & 715 & 100 & 349 & 100 & 961 & 100 \\
\hline 5 & - & - & 554 & 77 & 328 & 94 & 816 & 85 \\
\hline
\end{tabular}

- La période P9 cumule les facteurs défavorables : faible croissance hivernale, accumulation des épis, sénescence sans renouvellement des organes végétatifs et déssèchement final.

Mises à part les périodes P5 (juin-juillet) et P13 (mai à juillet) à la fin desquelles se produit le dessèchement, la productivité des périodes déterminées de 1, 2 ou 3 mois est soumise à la température, au déficit hydrique relatif et à la fertilité du milieu :

- Les basses températures de mars 84 ont un effet limitant sur les productivités des périodes P2 (mars) et P10 (mars-avril) dans les 4 stations.

- Un déficit hydrique relatif apparu dans la chênaie à oxycèdre en avril et mai 1983 a un effet limitant sur P3 (avril), P4 (mai), P10 (mars-avril) et $\mathrm{P} 12$ (avril-mai), un peu plus accentué sur les périodes de 2 mois.

- En l'absence d'une franche limitation par le froid ou la sécheresse, c'est-à-dire en 1984 aux 3 étages inférieurs, et en 1983 à l'étage de la pelouse asylvatique, pour $\mathrm{P} 3, \mathrm{P} 4, \mathrm{P} 12, \mathrm{P} 11$, les productivités font apparaître des différences de fertilité entre les 4 stations.

Dans l'ordre de fertilité croissante :

$$
\text { - la cédraie : } \quad 2,74 \mathrm{~kg} \mathrm{ha}^{-1} \mathrm{j}^{-1}
$$

- la chênaie verte à oxycèdre : 4,56

- la chênaie verte pure : $\quad 7,02$

- la pelouse sur basalte : $\quad 9,71$

A titre de comparaison, la productivité de base des pâturages du Massif central, sans fertilisation, mais bien pâturés, avec recyclage sur place des déjections, avoisine couramment 15 à $20 \mathrm{~kg}$ $\mathrm{ha}^{-1} \mathrm{j}^{-1}$ sur les sols basaltiques, soit $3 \mathrm{t}$ de M.S. en 150 jours (Jeannin et al., 1979), 10 à $15 \mathrm{~kg}$ $\mathrm{ha}^{-1} \mathrm{j}^{-1}$ sur les sols granitiques (de Montard, 1983), 5 à $10 \mathrm{~kg} \mathrm{ha}^{-1} \mathrm{j}^{-1}$ sur dolomie et sur calcaire des Causses (Langlet et al., 1979). C'est donc à cette dernière situation qu'on peut comparer les parcours du Moyen Atlas. La durée de la période, 1, 2 ou 3 mois, n'a ici pas d'influence mesurable en pleine saison de végétation.

Variation de la productivité du tapis herbacé en fonction de l'étage bioclimatique et des caractéristiques du sol

Variation de la productivité moyenne des périodes d'un mois, à l'échelle de la station (Tableau III)

Construction d'un modèle d'ajustement statistique Un modèle mathématique a été construit pour représenter les variations de productivité de 
la strate herbacée des périodes d'un mois, P2, P3, P4 et P5. II a été obtenu par régression multiple progressive et condensation des 6 variables élémentaires retenues en 2 variables élaborées de valeur explicative très supérieure :

\section{Equation 1}

M.S. = RgA.ET.Tm-1,4 (0,89 LG.Ke + 0,055 NLG.LG-1.Ke $\left.\mathrm{Ke}^{-1}\right)$

avec $r^{2}=0,645$ et $n=31$.

Dans cette équation :

- M.S. désigne la matière sèche herbacée récoltée en $\mathrm{kg} \mathrm{ha}^{-1} \mathrm{j}^{-1}$;

- RgA et ET sont respectivement le rayonnement absolu moyen journalier en cal $\mathrm{cm}^{-2} \mathrm{j}^{-1}$ et l'évapotranspiration de l'ensemble de la couverture végétale en $\mathrm{mm} \mathrm{j}^{-1}$; $E T$ résulte du calcul du bilan des pluies et de I'ETP, sur la base d'une réserve utile moyenne de $100 \mathrm{~mm}$;

- Tm est la température moyenne de la période;

- LG désigne la somme des abondances-dominances (méthode Braun-Blanquet) des légumineuses;

- Ke est la moyenne par station des teneurs en potassium échangeable de l'horizon supérieur du sol;

- NLG désigne l'abondance-dominance des nonlégumineuses.

Le terme constant n'étant pas significativement différent de zéro, l'équation a été recalculée sans terme constant. Les intervalles de confiance au seuil 0,95 des coefficients de régression sont respectivement : $0,89 \pm 0,24$ et $0,055 \pm 0,032$.

Le premier terme, (RgA.Tm-1,4.LG.Ke), couvre $53 \%$ de la variance totale de la productivité en M.S.; le second, (RgA.ET.Tm-1,4.NLG.LG ${ }^{-1} \cdot \mathrm{Ke}^{-1}$ ) en couvre $11,5 \%$.

Interprétation du modèle Des essais itératifs et systématiques de décomposition du modèle en ses variables composantes ont montré que le meilleur modèle additif qui puisse en être déduit implique les trois variables suivantes:

$$
\begin{aligned}
& \text { V1 }=\mathrm{ET} \cdot \mathrm{Tm}^{-1} \\
& \text { V2 }=\mathrm{LG} \cdot \mathrm{Ke} \cdot \mathrm{RgA} \cdot \mathrm{Tm}^{-0,4} \\
& \mathrm{~V}_{3}=\mathrm{NLG} \cdot \mathrm{LG}^{-1} \cdot \mathrm{Ke}^{-1} \cdot \mathrm{RgA} \cdot \mathrm{Tm}^{-0,4}
\end{aligned}
$$

Ce passage du modèle produit au modèle additif s'effectue au prix d'une baisse importante du taux de variance expliquée : $r^{2}=0,58$ seulement dans le second. Le premier résulte de la somme des deux interactions, V1.V2 et V1.V3.

La variable $\mathrm{V} 1$ a la signification d'une potentialité de production par station et par période, exprimée par le rapport de l'évapotranspiration à la température. Ce rapport exprime que la production de la strate herbacée est ici proportion- nelle à la quantité d'eau disponible pour l'ensemble de la végétation (Morizet et Robelin, 1984) et que son efficience diminue lorsque la température s'élève; cet effet de la température est lié à l'accroissement de la demande climatique pour l'eau (Bouchet, 1963) et à l'aggravation du stress hydrique lorsque le déficit relatif s'accroit.

La Figure 2 montre que les déficits importants ne sont apparus qu'aux températures supérieures à $17^{\circ} \mathrm{C}$, mais pas systématiquement. En dessous de ce seuil, et quelque soit l'étage considéré, ET est proche de ETP et proportionnel à la température moyenne $(E T=0,147 \mathrm{Tm}$; avec $r=0,848^{* *}$; terme constant non différent de zéro). Au-delà, elle est très variable, à l'image de l'irrégularité des pluies en fin de printemps.

Les variables V2 et V3 correspondent à deux groupes d'espèces de physiologie différente qui contribuent à la production de la strate herbacée : les légumineuses dont les bactéries des nodosités fixent l'azote atmosphérique, et l'ensemble des autres espèces qui dépendent entièrement de la fourniture d'azote minéralisé par le sol.

Le terme climatique ( $\mathrm{RgA} . \mathrm{Tm}^{-0.4}$ ) exprime un bilan complexe de croissance par rapport au rayonnement absolu et à la température : dans le contexte présent, ET est mieux valorisée avec un rayonnement fort et une température modérée. Ce contexte mérite d'être souligné en raison de l'affaiblissement de la liaison habituelle entre rayonnement et température obtenu ici par le jeu du gradient d'altitude $\left(r^{2}=0,331\right)$. En effet, tandis que $\mathrm{RgA}$ suit des variations saisonnières régulières, la température subit deux sources de variations, le rythme solaire et l'altitude. On peut

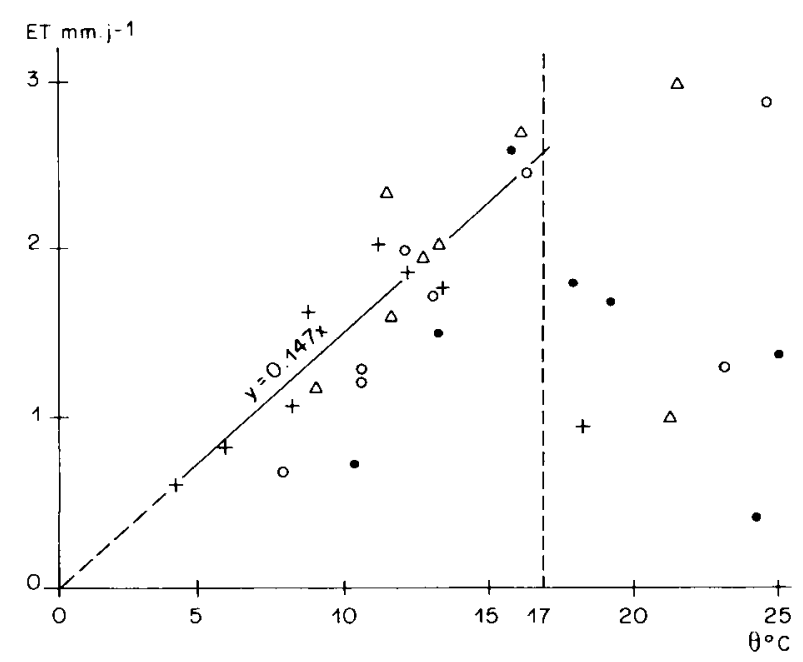

Fig. 2. Evapotranspiration (ET) calculée d'après le bilan hydrique : relation à la température $(\theta) ;(\bullet)$ Chênaie à oxycèdre; (o) Chênaie verte pure ; $(\Delta)$ Cédraie; (+) Pelouse asylvatique. 
rapprocher le rapport $\mathrm{RgA} . \mathrm{Tm}^{-0.4}$ du quotient photothermique (Durand, 1967; Fleury et Leterme, 1987).

La décomposition du modèle en $\mathrm{V} 2$ et $\mathrm{V} 3$, associant préférentiellement dans un modèle intermédiaire les deux groupes d'espèces à la variable $\left(\mathrm{RgA} . \mathrm{Tm}^{-0.4}\right)$, indépendamment du rapport ET.Tm-1, pourrait indiquer que la différenciation du compartiment physiologique des deux groupes d'espèces se fait principalement autour de la compétition pour la lumière. La nette supériorité du modèle complet (équation 1) montre toutefois qu'il faut absolument intégrer cette compétition pour la lumière dans une interaction avec l'alimentation en eau.

Le potassium échangeable du sol joue aussi un rôle différentiel entre les deux groupes d'espèces : d'une part, il affecte la contribution directe des légumineuses à la matière sèche et d'autre part, leur capacité de compétition avec l'ensemble des espèces non fixatrices : la contribution de celles-ci à la productivité est, en effet, en relation inverse avec le produit (LG.Ke).

Le groupe des légumineuses domine le fonctionnement de la strate herbacée; en effet, la variable d'interaction V1.V2 absorbe $53 \%$ de la variance totale. Si on isole le produit (LG.ET.Ke) dans le modèle, on constate qu'on rejoint les conclusions de Shamsun-Noor et al. (1989) concernant l'interaction positive "eau $x$ potassium" sur la fixation symbiotique; nos résultats permettent d'y adjoindre l'interaction de la lumière et de la température.

Discussion. Limitations du modèle L'abondance-dominance pourrait être rapprochée de la notion d'indice foliaire; mais, elle n'a ici d'intérêt qu'à titre d'indice global des volumes occupés respectivement par les deux groupes d'espèces, les relevés de végétation n'ayant été faits qu'une fois, au cours de la première année. L'énergie incidente n'est connue que grossièrement, d'après RgA, c'est-à-dire sans tenir compte de l'épaisseur de l'atmosphère, ni de la couverture nuageuse.

Un facteur de grande importance, le taux de couvert forestier n'est pas pris en compte; c'est l'une des raisons de la limitation à $65 \%$ de la variance expliquée. Cependant, l'extrême hétérogénéité des sols à l'échelle de la placette de récolte a été en partie estompée en regroupant par station les observations par sous-station : un nouveau développement du modèle intégrant l'effet du couvert arboré est donc souhaitable mais peu aisé.

A défaut de disposer d'un autre lot de données dans des sites analogues, la validation du modèle reste en suspens. Sa robustesse peut cepen- dant être éprouvée en considérant deux segments de la gamme des températures :

- entre 4 et $13^{\circ} \mathrm{C}$ l'équation finale retenue est :

(2) $M S=1,002$ ET.RgA.LG.Ke. Tm-1,4

avec $r^{2}=0,667$ et $n=15$; ce résultat montre qu'aux faibles températures et en l'absence de déficit hydrique, seule la contribution des légumineuses est significative;

- entre 13 et $25^{\circ} \mathrm{C}$ l'équation finale conserve les deux termes avec des coefficients 0,72 et 0,058 respectivement, mais seulement $34 \%$ de la variance est expliquée.

En l'appliquant aux différents étages bioclimatiques, le modèle se révèle médiocre à l'étage semi-aride (variance expliquée $32 \%$ ), bien adapté dans les domaines de la chênaie verte et de la cédraie (64\%); à l'étage humide très froid qui ne comporte qu'une sous-station, il ne peut être testé séparément.

De cette mise à l'épreuve, il ressort que la principale faille réside dans la difficulté d'évaluation de l'ET : la mesure de l'énergie incidente et celle de l'évolution de l'état d'humidité des sols est indispensable pour aller vers un modèle agrophysiologique.

D'un point de vue épistémologique, le cadre de l'expérimentation proposée révèle une grande puissance pour associer dans un modèle interactif cohérent un ensemble de variables actives statistiquement et choisies pour leur signification agrophysiologique.

Dans les expérimentations plus fines où la structure du couvert et les fonctions physiologiques sont suivies à des pas de temps très courts, les facteurs afférents sont plus couramment étudiés 2 à 2 seulement : en l'état, le modèle proposé peut aider à la formulation des hypothèses et à l'orientation des recherches plus approfondies, indispensables pour aller vers des modèles de fonctionnement écophysiologique intégrant les connaissances.

Facteurs affectant l'abondance-dominance des légumineuses Les légumineuses jouant un rôle dominant dans la productivité des parcours, il est nécessaire d'étudier leurs variations qui sont fortes d'un milieu à l'autre. Elles sont en faible abondance dans la chênaie à oxycèdre et la cédraie (LG de 0,5); assez abondantes dans la chênaie verte pure ( $L G=3,5)$, et intermédiaire dans la pelouse asylvatique $(L G=1,6)$. Une analyse par sous-station a permis de reconnaître comme facteurs actifs le taux de couvert arboré et la teneur en potassium échangeable du sol :

- la teneur du sol en potassium échangeable est toujours faible dans les sols sur schistes de l'étage de la chênaie à oxycèdre; 
- au taux de 20 à $60 \%$, le couvert arboré est favorable aux légumineuses; elles disparaissent sous couvert dense et sont peu abondantes à découvert. C'est précisément le cas de la station de la cédraie où des couverts moyens n'ont pas pu être échantillonnés.

A ces deux étages, la faiblesse des légumineuses, et la faible productivité qu'elle entraîne, seraient donc dues à des causes différentes, pauvreté en potassium pour l'un, et couvert arboré trop faible ou, au contraire, excessif pour l'autre. Pour les deux stations les plus riches en légumineuses, on ignore si l'intervention de la pâture autoriserait leur maintien en aussi grande abondance.

Variations de la productivité du tapis herbacé en fonction de l'étage bioclimatique et du couvert arboré (moyenne des périodes déterminées; Tableau VI)

A l'échelle de la sous-station, on observe l'effet des paramètres localisés, c'est-à-dire, le taux de couvert arboré, la situation topographique et les caractéristiques physiques et chimiques du sol.
Les mesures de productivité effectuées sous des couverts arborés inférieurs à 70 ou $80 \%$ (mesurés au densiomètre) mettent en évidence un phénomène tout à fait remarquable et de très forte conséquence pour la conception des aménagements sylvo-pastoraux du futur :

a) A l'étage du semi-aride, de la chênaie à oxycèdre :

- sur l'ensemble des sous-stations directement comparables $(4,5,7,2$ et 6$)$, le taux de couvert arboré n'a pas d'influence (année sèche) ou une influence légèrement favorable (année plus humide) dans la gamme allant de 0 à $62 \%$;

- parmi les autres, deux sous-stations pas ou peu couvertes ont une productivité très médiocre qui s'explique par un sol graveleux $(74 \%$ de sables grossiers) en exposition sud (sous-station 1) et par une position sommitale (sous-station 3);

- la sous-station 9, qui extériorise la meilleure productivité, occupe une situation favorable particulière, dans un vallonnement;

- au taux de $70 \%$, le couvert arboré a un effet franchement dépressif (sous-station 8).

Tableau Vla. Productivité en m.s. des périodes déterminées de 1, 2 ou 3 mois dans la semi-aride (chênaie verte à oxycèdre; $\mathrm{kg} \mathrm{ha}^{-1} \mathrm{j}^{-1}$ ).

\begin{tabular}{lllllllllll}
\hline S/Station & 1 & 4 & 5 & 9 & 7 & 3 & 2 & 6 & 8 & moyenne \\
Couvert \% & 0 & 0 & 0 & 0 & 13 & 19 & 33 & 62 & 70 & \\
1983 & & & & & & & & & & \\
1984 & 0,76 & 2,34 & 2,90 & 3,73 & 3,74 & 1,08 & 2,35 & 2,93 & 1,65 & 2,27 \\
& 1,50 & 2,84 & 2,15 & 5,66 & 2,76 & 1,56 & 3,76 & 3,30 & 1,50 & 2,78 \\
\hline
\end{tabular}

Tableau Vlb. Productivité en m.s. des périodes déterminées de 1, 2 ou 3 mois dans le subhumide (chênaie verte pure du Dir; kg ha-1 $\mathrm{j}^{-1}$ ).

\begin{tabular}{lrrrrrrrrr}
\hline S/Station \\
Couvert \% & 12 & 13 & 14 & 16 & 11 & 17 & 10 & 15 & moyenne \\
& 0 & 0 & 19 & 20 & 38 & 39 & 62 & 91 & \\
1983 & & & & & & & & & \\
1984 & 5,51 & 4,72 & 6,15 & 3,02 & 5,46 & 4,85 & 4,91 & 0,61 & 4,40 \\
& 8,71 & 5,85 & 7,32 & 4,52 & 3,95 & 3,45 & 5,37 & 0,36 & 4,94 \\
\hline
\end{tabular}

Tableau VIc. Productivité en m.s. des périodes de 1, 2 ou 3 mois dans l'humide froid (cédraie à sous-étage de chêne vert, s-st. 18 à 23) et dans l'humide très froid (pelouse asylv., s-station $24 ; \mathrm{kg} \mathrm{ha}^{-1} \mathrm{j}^{-1}$ ).

\begin{tabular}{lrrrrrrrrr}
\hline S/Station \\
Couvert \% & 21 & 23 & 20 & 18 & 19 & 22 & $\left.\begin{array}{l}\text { moyenne } \\
\text { cédraie }\end{array}\right]$ & 24 \\
& 0 & 0 & 65 & 70 & 75 & 83 & 0 \\
1983 & 2,06 & 2,19 & 2,25 & 2,55 & 4,89 & 1,00 & 2,49 & ] & 6,42 \\
1984 & 2,46 & 2,68 & 1,75 & 1,89 & 3,05 & 1,07 & 2,15 & ] & 5,21 \\
\hline
\end{tabular}


b) A l'étage du subhumide, de la chênaie verte pure :

- le taux de couvert jusqu'à $62 \%$ n'a pas d'influence en année sèche $\left(4,9 \mathrm{~kg} \mathrm{ha}^{-1} \mathrm{j}^{-1}\right)$, mais exerce une influence défavorable en année humide : la productivité est alors de $6,7 \mathrm{~kg} \mathrm{ha}^{-1}$ $\mathrm{j}^{-1}$ en moyenne pour les couverts inférieurs à $30 \%$ et de 4,3 pour les couverts arborés compris entre 35 et $62 \%$. Toutefois, les sous-stations 11 et 17, dominées au départ par Anthyllis vulneraria, peuvent avoir souffert en $2^{\mathrm{e}}$ année de la sensibilité de cette espèce aux coupes répétées;

- un couvert de $91 \%$ n'autorise qu'une productivité extrêmement faible (sous-station 15).

c) A l'étage de l'humide froid, de la cédraie à sous-étage de chêne vert :

- on dispose de 4 sous-stations comparables, 2 à découvert et 2 couvertes à 65 et $70 \%$ : la productivité est stable ou légèrement accrue par le couvert en année sèche $\left(2,2\right.$ et $2,4 \mathrm{~kg} \mathrm{ha}^{-1} \mathrm{j}^{-1}$ respectivement); en année humide, elle est plus élevée à découvert $(2,6)$ et plus basse sous couvert $(1,8)$ qu'en année sèche;

- la sous-station 19, sur sol profond, extériorise, malgré un recouvrement de $75 \%$, une productivité nettement plus élevée, mais variant selon la même tendance : c'est-à-dire, une productivité sous couvert plus élevée en année sèche qu'en année humide, soit 4,9 et $3,0 \mathrm{~kg} \mathrm{ha}^{-1} \mathrm{j}^{-1}$ respectivement;

- la sous-station 22 , avec un couvert de $83 \%$ atteint seulement $37 \%$ de la productivité moyenne des autres sous-stations de la cédraie.

Ainsi, aux trois étages considérés, avec une sécheresse relative plus ou moins accentuée (les deux années dans le semi-aride, la première année dans le subhumide et l'humide), la perte d'éclairement serait compensée par un meilleur bilan hydrique à l'abri des arbres (Ovalle, 1986; Joffre, 1987) : alors, la productivité n'est pas affectée par une couverture arborée allant jusqu'à 60 ou $70 \%$. Par contre, en année humide, l'alimentation en eau étant suffisante, la lumière devient facteur limitant (Lemoine et al., 1983); la productivité n'est plus qu'à $65 \%$ de la productivité mesurée à découvert, par défaut d'éclairement.

Quelle que soit l'année climatique, les couverts denses étudiés aux différents étages (70\% dans la chênaie verte à oxycèdre, $91 \%$ dans la chênaie verte pure, $83 \%$ dans la cédraie) ont un effet dépressif accentué sur la productivité du tapis herbacé.

La cédraie paraît moins étouffante que la chênaie puisqu'on obtient de bonnes productivités jusqu'à 70 ou $75 \%$ de couvert dans la première et jusqu'à $65 \%$ seulement dans la seconde; cette constatation cadre bien avec la densité respective du feuillage des deux espèces.

Une sylviculture conçue pour maintenir un recouvrement inférieur à $65 \%$ dans la chênaie et à $75 \%$ dans la cédraie devrait donc conserver, au minimum, les deux tiers de la ressource pastorale herbacée, et permettre la culture d'arbres de belle venue en densité modérée, produisant une quantité de bois à peine légèrement inférieure à la production des forêts denses.

\section{Conclusion}

Ces résultats permettent de retenir plusieurs enseignements de grand intérêt pour la conception des aménagements sylvo-pastoraux :

- pour bénéficier à plein des ressources pastorales herbacées de chacun des étages bioclimatiques, les animaux doivent être présents assez tôt et avec un chargement suffisant pour supprimer une grande partie des organes reproducteurs et stimuler ainsi la repousse d'organes végétatifs tout au long de la saison humide;

- une étude plus poussée sur la pérennité des espèces herbacées en fonction de l'intensité du pâturage, particulièrement des légumineuses qui ont constitué une des clés de la productivité mesurée dans cette expérimentation, serait maintenant nécessaire. On déterminera ainsi par étage et par saison un optimum de chargement compatible avec le renouvellement de la ressource herbacée;

- la conception d'une sylviculture productive associée à l'élevage est possible dans ces milieux par une méthode en peuplements espacés respectueuse de la ressource pastorale herbacée. La condition principale du succès n'est pas technique, mais humaine : intéresser positivement les pasteurs à la bonne conduite des arbres et prévoir un plan d'allégement des effectifs ovins sur le parcours dans les périodes et les années difficiles.

\section{Références}

Bouchet R.J. (1963) Evapotranspiration réelle, évapotranspiration potentielle et production agricole. Ann. Agron. 14, 743-824

Christie R.K. (1978) Ecosystem process in semi-arid grassland. Primary production and water status in two communities possessing different photosynthetic pathways. Austr. J. Agric. Res, 29, 773-787

Cornet A. (1981) Mesure de biomasse et détermination de la production aérienne nette de la strate herbacée dans la zone sahélienne du Sénégal. Acta CEcologica, CEcologia plant. 2, (3), 251-266

Durand R. (1967) Action de la température et du rayonnement sur la croissance. Ann. Physiol. Vég. 9, (1), 5-27 
Fleury A. \& Leterme P. (1987) Conditions d'utilisation du quotient photothermique pour le diagnostic cultural. C.R. Acad. Agric. Fr. 73, (2), 37-43

Gounot M. \& Yu O. (1980) Recherches sur l'évaluation de la productivité primaire épigée des graminées prairiales. Acta CEcologica. CEcologia Plant. 1, (3), 231-244 Jeannin B., Garel J.P., Louyot J., de Montard F. \& Petit M. (1979) Production et utilisation rationnelle des pâturages d'altitude dans les montagnes humides du Massif Central, pp. 137-155. In: Utilisation par les ruminants des pâturages d'altitude et des parcours méditerranéens. INRA

Joffre R. (1987) Contraintes du milieu et réponses de la végétation dans les dehesas de la Sierra Norte. Thèse, Montpellier

Langlet A., Flamant J.C., Molenat G. \& Osty P.L. (1979) Les parcours des Grands Causses : contraintes et possibilités techniques d'une mise en valeur par l'élevage ovin. In: Utilisation par les ruminants des pâturages d'altitude... 257-334, éd. INRA

Lemoine B., Bonhomme D., Chinzi D., Comps B., Bergeret H., Gelpe J., Juste C. \& Menet M. (1983) Elevage en forêt dans les Landes de Gascogne. I - Le système végétal. Ann. Sci. Forest. 40, (1), 3-40

Levang P. \& Grouzis M. (1980) Méthodes d'étude de la biomasse herbacée de formations sahéliennes : appli- cations à la Mare d'Oursi, Haute Volta. Acta CEcologica, CEcologia Plantarum 1, (3), 231-244

Milner C. \& Elfynhughes R. (1968) Methods for the measurement of the primary production of grassland; IBP Handbook, 6

Montard F.X. de (1983a) Climat et potentialités herbagères, 439-456; (1983b) Productivité herbagère des prés et des pacages en Margeride, 457-473. In: La Margeride, la montagne, les hommes INRA, Paris

Morizet J. \& Robelin M. (1984) Résultats de 18 années d'observations lysimétriques sous climat limagnais. II Etude des relations entre l'eau et la production végétale. Agronomie, 4, (5), 407-416

Ovalle C. (1986) Etude du système écologique sylvopastoral à Acacia Caven. Thèse université du Languedoc, Montpellier

Qarro M. (1985) Etude de la productivité du tapis herbacé des parcours de la zone d'Ain-Leuh au Maroc (Moyen Atlas-Plateau central). Thèse en Ecologie, Faculté St-Jérôme, Université Aix-Marseille

Saugier B. (1982) Ecosystème prairial. Photosynthèse et production; principe de modélisation et application à

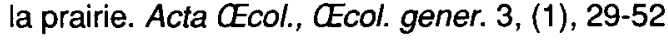

Shamsum-Noor L., Robin C., \& Guckert A. (1989) Effet d'un déficit hydrique sur le trèfle blanc. II - Rôle d'un apport de potassium présenté à Agronomie 